\title{
Left ventricular T2 distribution in Duchenne Muscular Dystrophy
}

\author{
Janaka P Wansapura ${ }^{1 *}$, Kan N Hor ${ }^{2}$, Wojciech Mazur ${ }^{3}$, Robert Fleck' ${ }^{1}$, Sean Hagenbuch², D Woodrow Benson ${ }^{2}$, \\ William M Gottliebson²
}

\begin{abstract}
Background: Although previous studies have helped define the natural history of Duchenne Muscular Dystrophy (DMD)-associated cardiomyopathy, the myocardial pathobiology associated with functional impairment in DMD is not yet known.

The objective of this study was to assess the distribution of transverse relaxation time (T2) in the left ventricle (LV) of DMD patients, and to determine the association of myocardial T2 heterogeneity to the severity of cardiac dysfunction. DMD patients $(n=26)$ and normal control subjects $(n=13)$ were studied by Cardiovascular Magnetic Resonance (CMR). DMD subject data was stratified based on subject age and LV Ejection Fraction (EF) into the following groups: $A(<12$ years old, $n=12) ; B(\geq 12$ years old, $E F \leq 55 \%, n=8)$ and $C(\geq 12$ years old, $E F=55 \%, n=$ 6). Controls were also stratified by age into Groups $N 1(<12$ years, $n=6)$ and N2 ( $>12$ years, $n=5)$. LV mid-slice circumferential myocardial strain $\left(\varepsilon_{\mathrm{cc}}\right)$ was calculated using tagged CMR imaging. T2 maps of the LV were generated for all subjects using a black blood dual spin echo method at two echo times. The Full Width at Half Maximum (FWHM) was calculated from a histogram of LV T2 distribution constructed for each subject.
\end{abstract}

Results: In DMD subject groups, FWHM of the T2 histogram rose progressively with age and decreasing EF (Group A FWHM= $25.3 \pm 3.8 \mathrm{~ms}$; Group B FWHM= $30.9 \pm 5.3 \mathrm{~ms}$; Group C FWHM= $33.0 \pm 6.4 \mathrm{~ms})$. Further, FWHM was significantly higher in those with reduced circumferential strain $\left(\left|\varepsilon_{c c}\right| \leq 12 \%\right)$ (Group B, and C) than those with $\left|\varepsilon_{c c}\right|$ $>12 \%$ (Group A). Group A FWHM was not different from the two normal groups (N1 FWHM $=25.3 \pm 3.5$ ms; N2 $F W H M=24.0 \pm 7.3 \mathrm{~ms}$ ).

Conclusion: Reduced EF and $\varepsilon_{c c}$ correlates well with increased T2 heterogeneity quantified by FWHM, indicating that subclinical functional impairments could be associated with pre-existing abnormalities in tissue structure in young DMD patients.

\section{Background}

Duchenne Muscular Dystrophy (DMD), a lethal Xlinked skeletal and cardiac myopathy, affects $1 / 3500$ males [1]. DMD is caused by mutations in the dystrophin gene resulting in greatly reduced or absent dystrophin protein, and is characterized by progressive skeletal muscle weakness, with loss of ambulation typically during the second decade [2,3]. A proposed mechanism of DMD pathogenesis in cardiac muscle is an alteration in calcium $\left(\mathrm{Ca}^{2+}\right)$ homeostasis, whereby micro-tears in the sarcolemmal membrane and non-specific channel opening events initiate a destructive cascade culminating in

\footnotetext{
* Correspondence: janaka.wansapura@cchmc.org

'Division of Radiology, Cincinnati Children's Hospital Medical Center, Cincinnati, Ohio, USA
}

myocyte necrosis and fibrosis [4-6]. Studies have shown that occult ventricular dysfunction and myocardial fibrosis can be diagnosed by Cardiovascular Magnetic Resonance (CMR) in DMD $[7,8]$. Previously, we found that peak left ventricular composite myocardial circumferential strain $\left(\varepsilon_{\mathrm{cc}}\right)$ is reduced early in the course of DMD despite normal ejection fraction (EF), and the strain values continue to decline with advancing age[9]. While this and other studies have helped define the natural history of DMD-associated cardiac dysfunction by characterizing changes in myocardial mechanics, assessment of the myocardial pathobiology associated with the regional functional impairment in DMD has not been described. As such, an index of DMD-associated cardiac dysfunction that defines the pathobiology of the disease

\section{Biomed Central}


would enhance our ability to assess the natural history of this disease.

A CMR methodology for evaluating in-vivo pathobiology has, however, been available for quite some time. The measure of transverse relaxation time (T2) of tissue has been well-characterized; T2 weighted MR signal has been used to detect various pathologies of soft tissues $[10,11]$. Factors such as fatty infiltration [12], increased water content and vasogenic edema[13] are known to increase $\mathrm{T} 2$, while collagen accumulation, as seen in extracellular matrix fibrosis, is known to decrease T2 [14]. In recent years, T2 weighted imaging, as well as absolute T2 mapping, has been demonstrated to be valuable in cardiac imaging of acute myocardial process such as myocardial infarct [15-17], myocarditis [18,19], and acute cell mediated transplant rejection[20].

DMD is an example of a progressive disease process where fibrosis and edema related to inflammation coexist. In a previous study conducted at a magnetic field strength of 0.5 Tesla, Mavrogeni et al [21] showed that DMD patients had lower cardiac T2 relaxation time which progressively decreased with age compared to control subjects. The same authors recently showed that with steroid treatment, myocardial T2 increased in older DMD patients [22]. However, Mavrogeni et al [21] limited their consideration to the mean $\mathrm{T} 2$ value in the septal region of the heart, forgoing analysis of T2 distribution across multiple regions of the LV. However, we and others have shown $[23,24]$ that myocardial fibrosis and regional abnormalities appear heterogeneously, with initial appearance of abnormalities typically in the LV free wall.

We thus hypothesized that heterogeneity of $\mathrm{T} 2$ across an entire slice of the heart exist in the early stages of DMD-associated cardiomyopathy and are associated with the subclinical functional abnormalities quantified by myocardial circumferential strain. The purpose of this study was thus to evaluate the heterogeneity of $\mathrm{T} 2$ distribution in the LV in boys with various stages of DMD.

\section{Methods}

\section{Study population}

Data were analyzed from records of DMD subjects followed at Cincinnati Children's Hospital Medical Center. The diagnosis of DMD was confirmed by skeletal muscle biopsy showing absent dystrophin and/or DNA analysis demonstrating a characteristic dystrophin mutation in all patients. DMD patients who underwent clinical CMR between September 2005 and September 2007 were included in this analysis. Additionally, two control groups of young (N1, $\mathrm{n}=8$, age 5-10 years) and older $(\mathrm{N} 2, \mathrm{n}=5$, age $32-45$ years) normal control males underwent an identical protocol. EF and $\left|\varepsilon_{\mathrm{cc}}\right|$ measurements were not available in the N2 control group. The Institutional Review Board at the Cincinnati Children's Hospital Medical Center approved the study.

All subjects were $>5$ years of age, thereby eliminating the need for sedation. CMR was performed at our center on 97 DMD patients between September 2005 and September 2007. Data from 71 was excluded due to absence of T2 images $(n=44)$, absence of tagged images $(\mathrm{n}=18)$ or poor tag quality secondary to breathing artifact or patient movement $(\mathrm{n}=9)$.

\section{Subject stratification}

The subject data were stratified into 5 groups (two groups of control subjects: $\mathrm{N} 1(\mathrm{n}=6)$ and $\mathrm{N} 2(\mathrm{n}=5))$ and three groups of DMD patients $(A(n=12), B$ $(n=8)$, and $C(n=6))$. Stratification in the control subjects was based on age. In the DMD subjects stratification was primarily by age and secondarily by LV Ejection Fraction (EF) (Figure 1). Thus, Group A comprised DMD subjects age $<12$ years with normal EF. Group B were DMD subjects age $\geq 12$ years with normal EF. Group C included DMD subjects age $\geq 12$ years with reduced EF $(\leq 55 \%)$. Due to the significant dichotomy in ages of the control subjects, they were also stratified by age into Groups N1 ( $<12$ years) and N2 ( $>12$ years), due to the significant dichotomy in ages of these subjects.

\section{CMR acquisition}

CMR was performed on a Siemens 3-T Trio (Siemens Medical Solutions, Malvern, Pennsylvania/Erlangen, Germany) system.

\section{Imaging protocols \\ Ventricular Volumetry and Global Functional Imaging}

Cardiac functional imaging was performed as previously described [9]. CMR was acquired with retrospective electrocardiogram (ECG)-gating, segmented steady-state free precession technique after localized shimming and/ or frequency adjusting. Subjects were breath-held as tolerated; for those subjects who could not adequately breath-hold, a free breathing technique with multiple signal averaging was used. Standard imaging included a short-axis stack of cine SSFP images from cardiac base to apex.

\section{Myocardial Strain Imaging}

Tagged cine CMR were acquired in the short axis of the mid left ventricle at the level of the papillary muscles with an ECG-triggered segmented k-space fast gradient echo sequence with spatial modulation of magnetization in orthogonal planes. Grid tag spacing was 7 to $8 \mathrm{~mm}$. The scan parameters used were: field of view $(30-32) \times$ (25 - 26) $\mathrm{cm}^{2}$, slice thickness $6 \mathrm{~mm}$, flip angle $20^{\circ}$, TE/ TR $3 \mathrm{~ms} / 4.2 \mathrm{~ms}$, views/segment 7-9. 


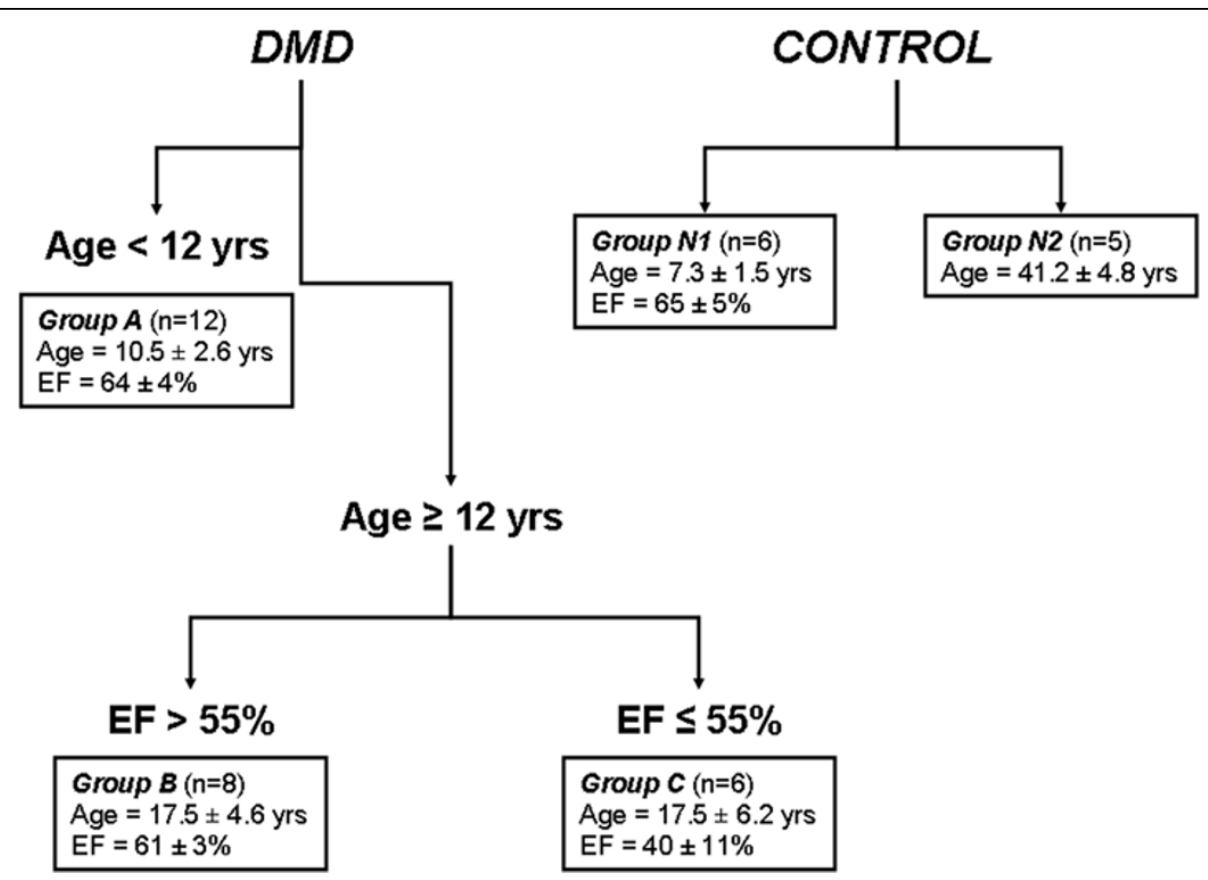

Figure 1 Categorization of the study population. Stratification of Duchenne muscular dystrophy (DMD) patients based on age and ejection fraction (EF). Normal controls were stratified according to age given their dichotomous distribution.

\section{T2 Imaging}

For T2 measurements, spin echo images of the left ventricle in the short axis plane were acquired using a black blood dual spin echo method. Imaging parameters were: Slice thickness $=5 \mathrm{~mm}$, in plane resolution $=1.4 \mathrm{~mm} \times$ $1.4 \mathrm{~mm}$, echo train length $=5$, Echo times: $\mathrm{TE}_{1}=6 \mathrm{~ms}$, $\mathrm{TE}_{2}=34 \mathrm{~ms}$. Data acquisition was done during late diastole by inserting a subject dependent delay.

\section{Data analysis}

\section{Ventricular Volumetry and EF}

Ventricular volumes, mass, and global function were assessed via standard planimetry techniques with semiautomated computer software (QMASS version 6.1.5, Medis Medical Imaging Systems, Leiden, the Netherlands) by expert readers (R.J.F., W.M.G., and K.N.H.).

\section{Myocardial strain analysis}

Tagged images were analyzed with the HARmonic Phase (HARP, Diagnosoft, Palo Alto, California) technique, as previously described [9]. Based on our experience and that of others regarding limited reproducibility of the basal and apical slices, only the midventricular slice was analyzed [8]. An average of all the regional values/subject was calculated as a composite whole-slice strain value $\varepsilon_{\mathrm{cc}}$, to allow for comparison purposes. All HARP strain analyses were performed by an experienced reader (K.N.H.). To assess inter-observer variability of HARP analysis, a second experienced reader (W.M.G) performed the same analysis on a subset of DMD subjects $(n=6)$ and controls $(n=3)$.

\section{T2 Analysis}

T2 values were calculated on a pixel-by-pixel basis according to the standard description of the signal behavior in spin echo magnetic resonance imaging as:

$$
\mathrm{T} 2=\left(\mathrm{TE}_{2}-\mathrm{TE}_{1}\right) / \operatorname{In}\left(\mathrm{S}_{1} / \mathrm{S}_{2}\right)
$$

where $S_{1}$ and $S_{2}$ are the signal intensity at echo times $\mathrm{TE}_{1}$ and $\mathrm{TE}_{2}$ respectively. Regions of interests were manually drawn (J.P.W.) to extract the T2 values. The LV was defined on the T2 weighted magnitude image (i.e. at $\mathrm{TE}=\mathrm{TE}_{2}$ ) by manually drawing (J.P.W) the endo- and epicardial boarders with care taken to avoid signal from blood. Similarly, lateral wall and septum was segmented manually as defined by the American Heart Association 17-segmental model i.e. septum $=$ regions 7 and 8; lateral wall $=$ regions 11 and 12 . Using these $\mathrm{T} 2$ values, a histogram of LV T2 distribution with bin size equal to $1 \mathrm{~ms}$ was constructed for each subject.

\section{FWHM Calculation}

For each histogram, the Full Width at Half Maximum $(F W H M)$ was calculated after applying box car averaging. The FWHM was defined as the width of the histogram at half the maximum height. Additionally, the mean and the standard deviation of $\mathrm{T} 2$ of the entire LV as well as in the septum and in the lateral wall were 
also calculated. All image processing related to T2 measurements were done using custom developed software written in the Interactive Data Language (IDL, Research Systems Inc., CO, USA).

\section{Statistical analyses}

Analyses were performed using $\mathrm{SAS}^{\circledast}$ (Version 9.2 Cary, NC). The General Linear Model procedure was used to access group differences among the means for FWHM adjusting for age. Since age was not shown to be significant, it was removed from the model. The distribution of T2 was slightly skewed; therefore the Wilcoxon Rank sum test was used. All tests were two-sided and p-values $\leq 0.05$ or confidence intervals that did not include zero were considered statistically significant.

\section{Results}

A summery of results is presented in Table 1.

EF and $\varepsilon_{\mathrm{cc}}$ Data

All DMD subjects had abnormal $\left|\varepsilon_{\mathrm{cc}}\right|<16 \%$, consistent with our prior work [9], despite normal EF in Groups A and $B$. As previously found, the mean $\left|\varepsilon_{\mathrm{cc}}\right|$ was significantly different among the DMD groups and progressively decreased with age and EF. Control subjects in group N1 had normal EF and normal $\left|\varepsilon_{\mathrm{cc}}\right|$ (>16\%).

\section{T2 Data}

Figure 2 shows the black blood T2 images acquired at echo times of $6 \mathrm{~ms}$ (Figure 2a) and $34 \mathrm{~ms}$ (Figure 2b) in a DMD subject. The T2 maps show heterogeneity of LV T2 distribution in a DMD patient (Figure 2d) compared to that of a normal subject (Figure 2c) and were quantified using T2 histograms (Figure 3). A Wilcoxon Rank Sum test showed no significant difference in the mean T2 of the entire LV among all groups. Similarly no significant difference in the standard deviation of $\mathrm{T} 2$ was found between groups. The age was not a significant factor in this model. However, the FWHM of the T2 histogram was significantly $(\mathrm{p}<0.05)$ greater in Groups $\mathrm{B}(30.9 \pm 5.3 \mathrm{~ms})$ and $\mathrm{C}(33.0 \pm 6.4 \mathrm{~ms})$ compared to that of Group A $(25.3 \pm 3.8 \mathrm{~ms})$ and the normal Groups
$\mathrm{N} 1(25.3 \pm 3.5 \mathrm{~ms})$ and N2 (24.0 $\pm 7.3 \mathrm{~ms})$ (Figure 4). This indicates that significantly greater $\mathrm{T} 2$ heterogeneity in Groups B and C with advanced cardiac dysfunction. These results also show that DMD patients with normal EF but impaired circumferential strain $\left(\left|\varepsilon_{\mathrm{cc}}\right| \leq 12 \%\right.$, Group B) had a significantly greater T2 heterogeneity than that of DMD patients with normal EF and $\left|\varepsilon_{\mathrm{cc}}\right|$ $>12 \%$ (Group A).

Among DMD patients with Normal EF (i.e. Groups A and B), a trend towards increasing FWHM with decreasing mean T2 of the LV (T2mean) was noticed. We characterized this trend by calculating the ratio FWHM/ T2mean for all subjects of groups A and B. FWHM/ T2mean was significantly $(\mathrm{p}<.0001)$ higher in group $\mathrm{B}$ $(0.54 \pm 0.08)$ compared to Group A $(0.42 \pm 0.06)$. Regression analysis showed moderate association between $F W H M /$ mean $\mathrm{T} 2$ and $\left|\varepsilon_{\mathrm{cc}}\right|$ in group $\mathrm{A}$ and $\mathrm{B}$ (Figure 5). (Pearson correlation coefficient $\mathrm{r}=.51$ ). Age alone was not found to be a significant factor in predicting T2 heterogeneity.

Results of regional analysis of mean T2, T2 FWHM and $\varepsilon_{\mathrm{cc}}$ are shown in Table 2. Mean T1 and T2 FWHM were higher in the septum than in the lateral wall. No difference in the septum and lateral wall was found in $\varepsilon_{\mathrm{cc}}$. Although not statistically significant the lateral wall T2 FWHM followed the same trend as T2 FWHM of the entire LV: Group A $(19 \pm 6 \mathrm{~ms})<$ Group B $(21 \pm 8$ $\mathrm{ms})<$ Group C $(26 \pm 6 \mathrm{~ms})$.

Three investigators blind to the identity of the subjects independently measured the mean T2 and T2 FWHM of 6 subjects using the custom made software. For each subject, the mean and the standard deviation of the independent measurements were calculated. The interobserver variability of T2 and T2 FWHM was assessed as the standard deviation/mean \%, averaged among all the subjects. The mean percentage of standard deviation among the investigators was $3.2 \%$ for meant $\mathrm{T} 2$ and $3.1 \%$ for T2 FWHM.

\section{Discussion}

In this study, we used the Full Width of Half Maximum (FWHM) of $\mathrm{T} 2$ distribution in LV to quantify the

Table 1 Summary of CMR findings between groups. Group values are presented as mean \pm standard deviation

\begin{tabular}{llllll}
\hline Group & EF $\%$ & $\mid$ Ecc| \% & Age & T2 (ms) & FWHM (ms) \\
\hline$A(n=12)$ & $64 \pm 4$ & $14.2 \pm 0.8^{*}$ & $10.5 \pm 2.6^{*}$ & $61 \pm 9$ & $25.3 \pm 3.8$ \\
\hline$B(n=8)$ & $60 \pm 3$ & $11.0 \pm 0.8 t^{*}$ & $17.5 \pm 4.6 t^{*}$ & $58 \pm 9$ & $30.9 \pm 5.3 t^{*}$ \\
\hline$C(n=6)$ & $40 \pm 11 \dagger \neq$ & $7.7 \pm 0.3 t^{*}$ & $17.5 \pm 6.3 t^{*}$ & $63 \pm 6$ & $33.0 \pm 6.4 t^{*}$ \\
\hline N1 $(n=6)$ & $65 \pm 5$ & $20 \pm 2.5$ & $7.3 \pm 1.6$ & $57 \pm 9$ & $25.5 \pm 3.5$ \\
\hline N2 $(n=5)$ & & $41.2 \pm 4.2$ & $46 \pm 11$ & $24.0 \pm 7.3$ \\
\hline
\end{tabular}

* The mean is statistically different to that of Group N1 with a $p<0.05$

† The mean of Group A is statistically different to that of Group B or C with a $p<0.05$

\# The mean of Group B is statistically different to that of Group C with a $p<0.05$ 

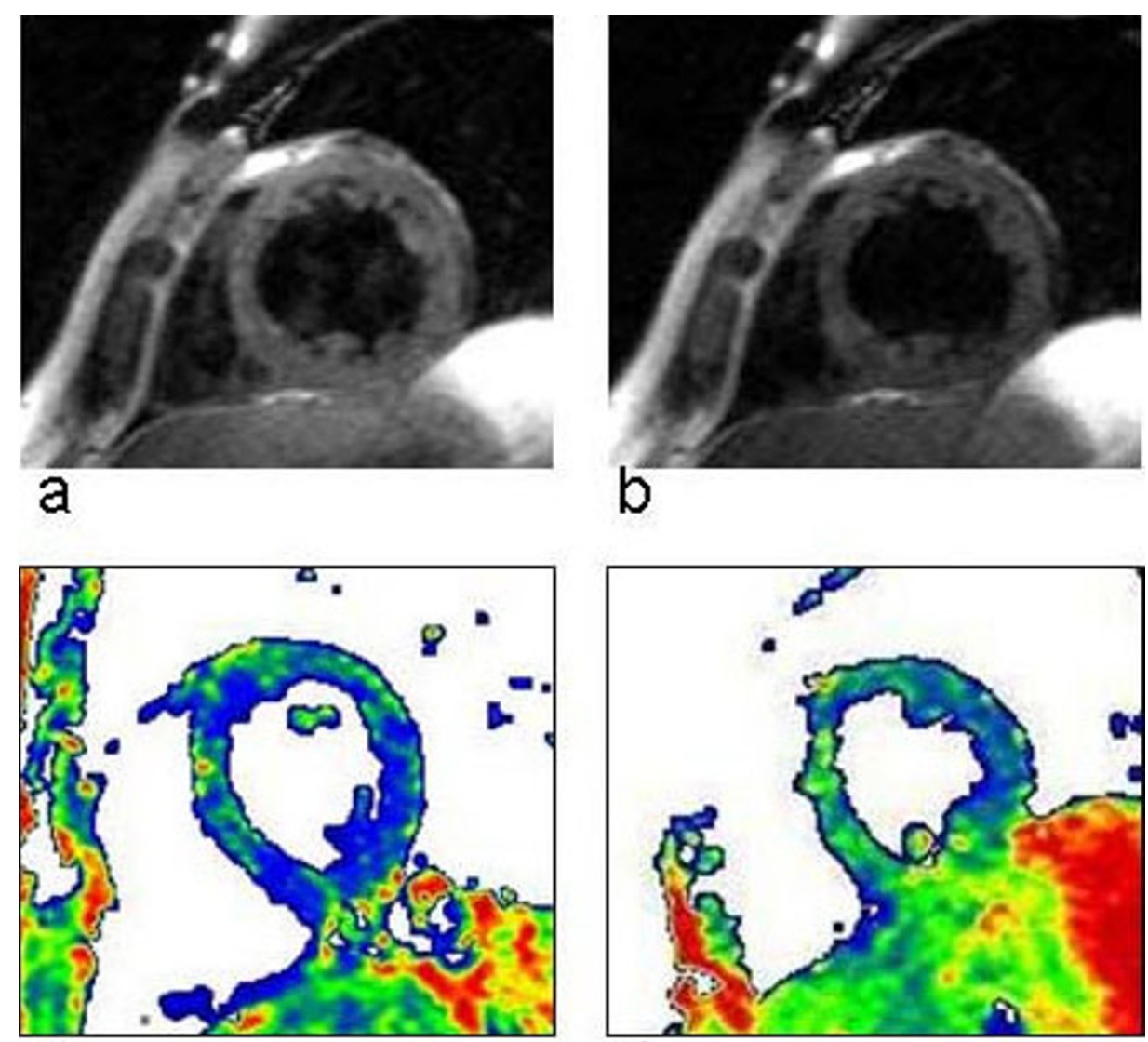

C
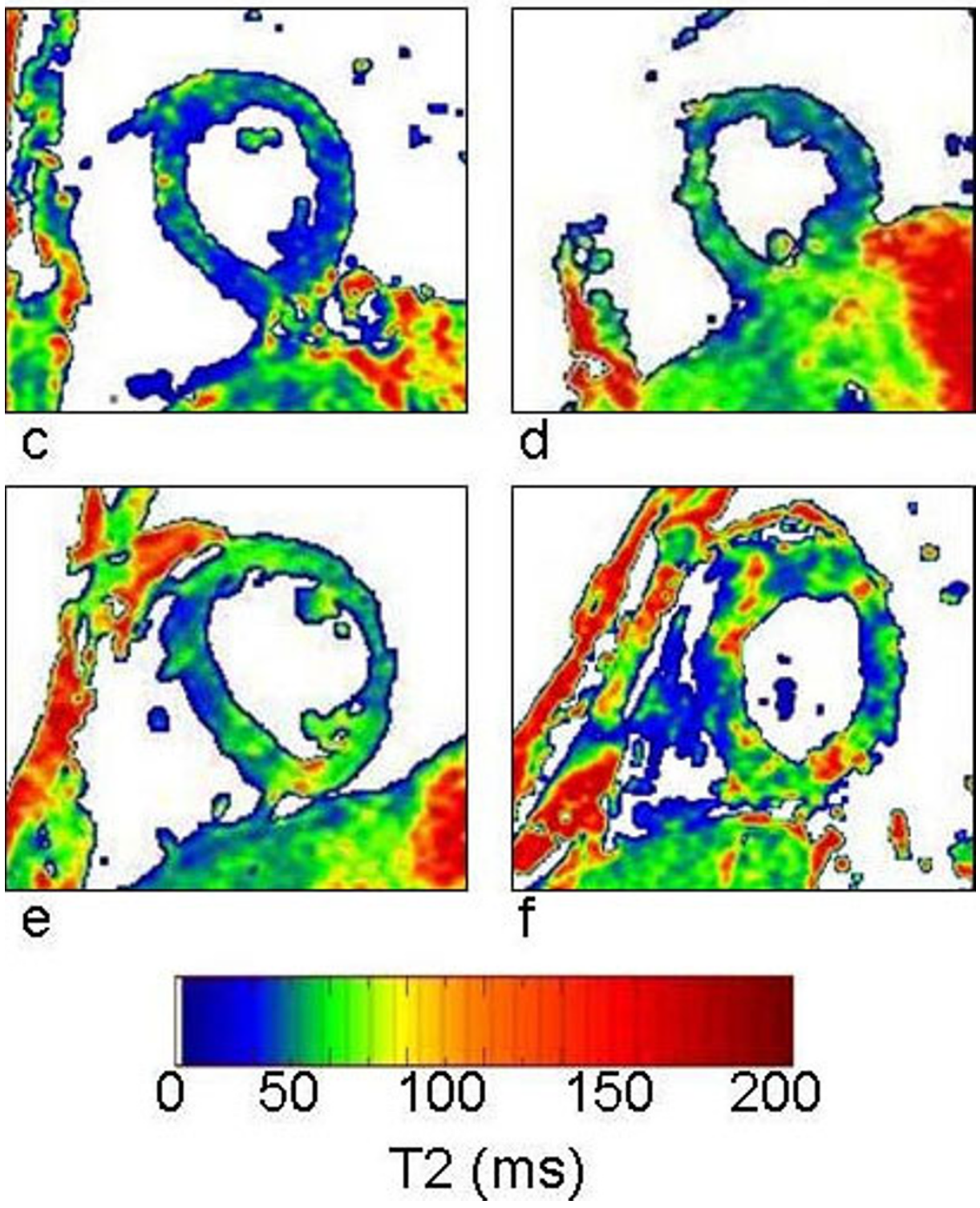

Figure 2 Left Ventricular T2 Maps. Black blood fast spin echo images of the left ventricle at TE $=6 \mathrm{~ms}(\mathrm{a})$ and TE= $34 \mathrm{~ms}$ (b) in the short axis view of a DMD subject. The T2 maps of a normal (c) and DMD subjects (d: group A, e: group B and f: group C) show the increasing heterogeneity of T2 in the LV corresponding to the severity of disease. 


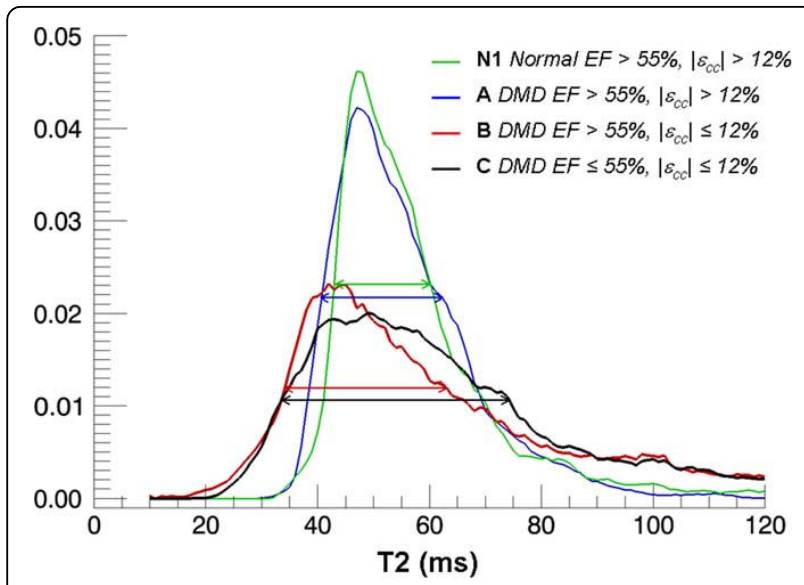

Figure 3 T2 histograms. Examples of normalized (i.e. area under curve $=1$ ) T2 histograms of DMD (Group A, B and C) and Normal control (N1) subjects show that DMD patients with normal EF but impaired $\varepsilon_{\mathrm{cc}}$ has higher heterogeneity in T2 compared to other groups.

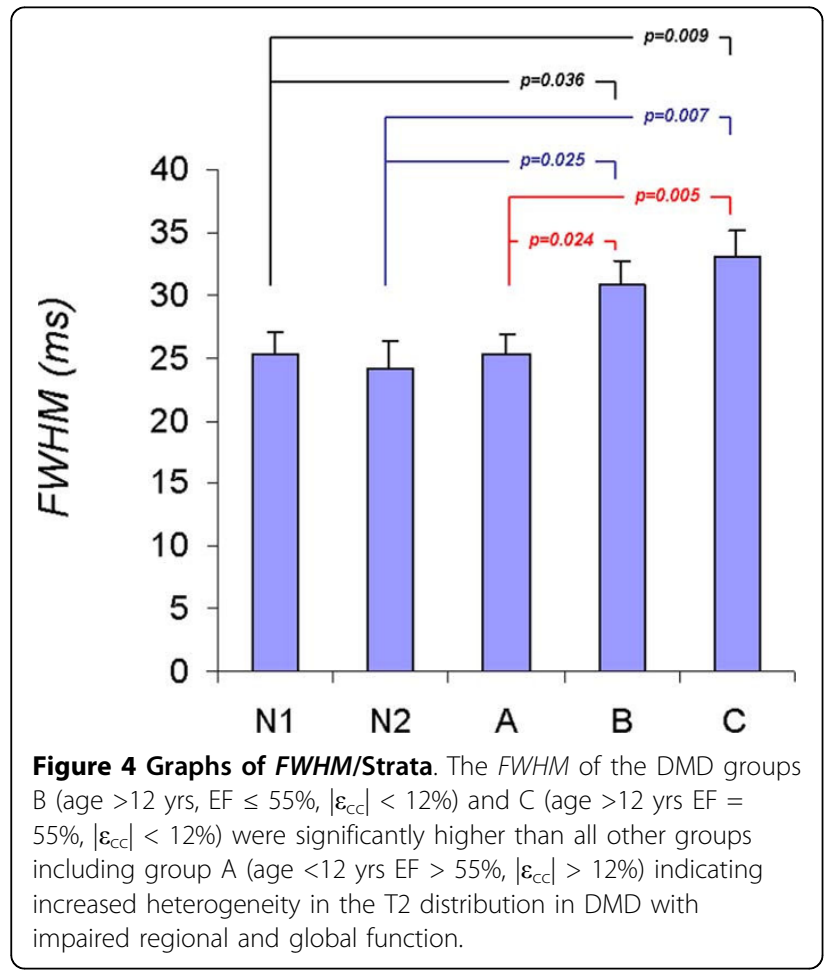

myocardial structural heterogeneity in DMD patients. In DMD subject groups, FWHM of the T2 histogram rose progressively with age and decreasing EF. Further, FWHM was significantly higher in those with reduced circumferential strain $\left(\left|\varepsilon_{\mathrm{cc}}\right| \leq 12 \%\right)$ (Group B, and C) than those with $\left|\varepsilon_{\mathrm{cc}}\right|>12 \%$ (Group A). The myocardial structural abnormality suggested by the observed trend is likely due to concomitant presence of micro-fibrosis (long before it becomes overt enough to be detected

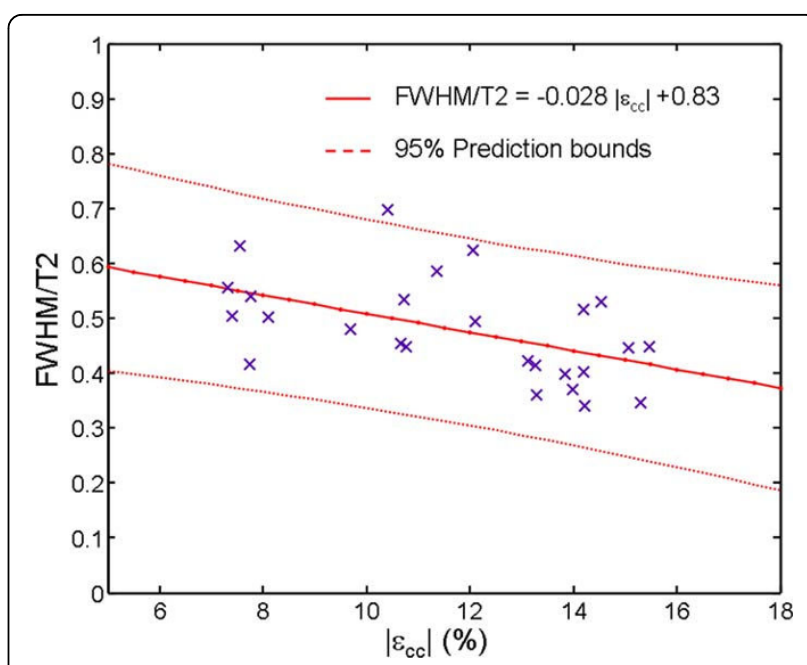

Figure 5 Regression of $\boldsymbol{F W H M / T 2 m e a n}$ vs $\boldsymbol{\varepsilon}_{\mathrm{cc}}$. Regression analysis show a moderate association between FWHM/T2mean and $\varepsilon_{c c}$ in group $A$ and $B$ (Pearson correlation coefficient $r=.51$ ) suggesting the presence of microscopic fibrosis long before it becomes overt enough to be detected by the LGE technique.

Table 2 Regional CMR findings between groups. Group values are presented as mean \pm standard deviation

\begin{tabular}{lllllll}
\hline & \multicolumn{3}{c}{ Mean T2 (ms) } & \multicolumn{2}{c}{ FWHM T2 (ms) } & \multicolumn{2}{c}{$\mid$ Ecc| (\%) } \\
\cline { 2 - 7 } & Septum & Free Wall & Septum & Free Wall & Septum & Free Wall \\
\hline A & $66 \pm 10$ & $55 \pm 12^{*}$ & $27 \pm 8$ & $19 \pm 6^{*}$ & $14.8 \pm 0.7$ & $14.7 \pm 0.8$ \\
\hline B & $63 \pm 13$ & $53 \pm 6$ & $27 \pm 10$ & $21 \pm 8$ & $11.2 \pm 1.5$ & $12.0 \pm 0.9$ \\
\hline C & $63 \pm 13$ & $66 \pm 12$ & $22 \pm 7$ & $26 \pm 6$ & $7.0 \pm 0.7$ & $7.7 \pm 0.9$ \\
\hline N1 & $67 \pm 20$ & $48 \pm 8^{*}$ & $22 \pm 8$ & $20 \pm 7$ & $16.8 \pm 1.0$ & $17.6 \pm 1.1$ \\
\hline N2 & $59 \pm 24$ & $43 \pm 9$ & $23 \pm 16$ & $19 \pm 10$ & - & - \\
\hline
\end{tabular}

by the myocardial Late Gadolinium Enhancement (LGE) technique) and myocardial edema. Our results support the notion that the regional dysfunction depicted by depressed $\left|\varepsilon_{\mathrm{cc}}\right|$ is associated with the ultrastructural myocardial cell abnormality present in DMD patients.

T2 is known to increase with intramuscular edema and inflammation that increases free water content in tissue [13] whereas fibrosis tend to decrease T2 relaxation time through increase in the collagen fractional area $[14,25]$. Autopsy studies have clearly shown that fibrosis and intramuscular edema co-exist in the DMD cardiac muscle [26]. Therefore the spread of T2, quantified by FWHM rather than the mean T2 is likely to better characterize this disease. Furthermore, LGE studies in muscular dystrophy show that distribution pattern of late enhancement is patchy and diffused unlike the well defined sub-endocardial LGE related to coronary territories found in myocardial infarction [7,24,27-29] signifying the spatial heterogeneity of the disease. The T2 
FWHM proposed in this study quantify both spatial and pathologic heterogeneity of the DMD tissue.

Unlike the FWHM, the standard deviation of T2, calculated assuming that T2 is normally (Gaussian) distributed within the LV, was not significantly different between groups. This is not surprising since the histograms of T2, as seen in Figure 3, deviate significantly from that of a normal distribution. The Skewness, a measure of deviation from the normal distribution, was $>1.2$ for all subjects confirmed this observation. Therefore, we conclude that the standard deviation of $\mathrm{T} 2 \mathrm{did}$ not accurately quantify the heterogeneity of $\mathrm{T} 2$ in the LV and consequently failed to distinguish DMD and normal subjects.

The mean myocardial T2 of the normal subjects (both $\mathrm{N} 1$ and N2 combined) in our study was $52 \pm 11 \mathrm{~ms}$ which is same as that found by Giri et al $(52 \pm 3 \mathrm{~ms})$ [30]. Regional analysis of T2 revealed that mean T2 was consistently lower in the lateral wall than in the septum in all groups. This may be due to the increased magnetic field inhomogeneity at the lateral wall due to the air-tissue interface. Increased B1 and B0 inhomogeneities at 3 Tesla pose significant challenges to uniform inversion of magnetization across the imaged volume affecting the accuracy of $\mathrm{T} 2$ calculations. As a routine practice $3 \mathrm{D}$ volume shimming should be employed to improve B0 homogeneity at 3 Tesla. Adiabatic radio frequency pulses, characterized by simultaneous modulation of amplitude and frequency or phase have been successful in mitigating B1 and B0 inhomogeneities [31-33]. These adiabatic pulses could be used in combination with $\mathrm{T} 2$ preparation schemes to generate differently weighted T2 images which then could be used to quantify T2.

\section{Study limitations}

We used a turbo spin echo method with two acquisitions (echoes) to calculate T2. The dual echo method has been successfully used by others in cardiac and brain imaging applications in the past $[13,30,34]$. While it allows image acquisition to be performed during a single breath hold, it limits the accuracy of the curve fitting. However, the alternative technique of acquiring multiple echo times requires scanning to be performed during free breathing leading to volume averaging of the signal that could also affect the accuracy of the T2. Giri et al[30] recently demonstrated the superiority of T2 prepared dual echo steady state free precision (SSFP) method for measuring cardiac T2 at 1.5 Tesla. With care taken to circumvent the effects of B0 inhomogeneity, this method could be ideally suited for cardiac T2 measurement in the DMD at 3Tesla.

Although significant differences in $\left|\varepsilon_{\mathrm{cc}}\right|$ and $F W H M$ were demonstrated between young DMD patients with normal EF and older patients with reduced EF, this is a cross-sectional and not a serial study. Accordingly, repeat serial examinations would provide a more robust analysis of longitudinal $\left|\varepsilon_{\mathrm{cc}}\right|$ and $F W H M$ in this patient population. In the current study, only the midventricular slice was analyzed secondary to our experience of limited reproducibility of the basal and apical slices.

\section{Conclusions}

The increased $F W H M$ in $\mathrm{T} 2$ points to an underlying pathobiology that is both microscopic and widely distributed within the myocardium. Unlike gross myocardial changes that arise in the later stages of the disease, these early abnormalities are not likely to be detected by T2 weighted imaging or LGE. Thus, T2 heterogeneity quantified by $F W H M$ could provide an early surrogate marker for pathobiology in DMD patients to monitor activity of disease as well as to assess efficacy of pharmacological interventions.

\section{Abbreviations}

DMD: Duchenne Muscular Dystrophy; T2: Transverse Relaxation Time; $\varepsilon_{c c}$ : Myocardial Circumferential Strain; EF: Ejection Fraction; $\mathrm{S}_{1}, \mathrm{~S}_{2}$ : Signal Intensity of Spin Echo Images; $\mathrm{TE}_{1}, \mathrm{TE}_{1}$ : Echo Times; FWHM: Full Width at Half Maximum; T2mean: mean T2 time of the Left ventricle; BO: Bore magnetic field strength; B1: Radio frequency transmit magnetic field strength.

\section{Author details}

'Division of Radiology, Cincinnati Children's Hospital Medical Center, Cincinnati, Ohio, USA. 'Division of Cardiology, Cincinnati Children's Hospital Medical Center, Cincinnati, Ohio, USA. ${ }^{3}$ Division of Cardiology, The Ohio Heart and Vascular Center, Christ Hospital, Cincinnati, Ohio, USA.

\section{Authors' contributions}

JW, KH, and WG contributed to all aspects of the manuscript's conception, design, data analysis, collection, critical revision and final approval. SH contributed to inter-observer variability analysis. WB, WM and RF contributed in data analysis, critical revision and final approval of the manuscript.

\section{Competing interests}

The authors declare that they have no competing interests.

Received: 4 November 2009 Accepted: 18 March 2010 Published: 18 March 2010

\section{References}

1. Moser H: Duchenne muscular dystrophy: pathogenetic aspects and genetic prevention. Hum Genet 1984, 66:17-40.

2. Bonilla E, Samitt CE, Miranda AF, Hays AP, Salviati G, DiMauro S, Kunkel LM, Hoffman EP, Rowland LP: Duchenne muscular dystrophy: deficiency of dystrophin at the muscle cell surface. Cell 1988, 54:447-452.

3. Perloff JK, de Leon AC Jr, O'Doherty D: The cardiomyopathy of progressive muscular dystrophy. Circulation 1966, 33:625-648.

4. Basset O, Boittin FX, Cognard C, Constantin B, Ruegg UT: BCl-2 overexpression prevents calcium overload and subsequent apoptosis in dystrophic myotubes. Biochem J 2006, 395:267-276.

5. Constantin B, Sebille $S$, Cognard C: New insights in the regulation of calcium transfers by muscle dystrophin-based cytoskeleton: implications in DMD. J Muscle Res Cell Motil 2006, 27:375-386.

6. Deconinck N, Dan B: Pathophysiology of duchenne muscular dystrophy: current hypotheses. Pediatr Neurol 2007, 36:1-7.

7. Silva MC, Meira ZM, Gurgel Giannetti J, da Silva MM, Campos AF, Barbosa Mde M, Starling Filho GM, Ferreira Rde A, Zatz M, Rochitte CE: Myocardial 
delayed enhancement by magnetic resonance imaging in patients with muscular dystrophy. J Am Coll Cardiol 2007, 49:1874-1879.

8. Ashford MW Jr, Liu W, Lin SJ, Abraszewski P, Caruthers SD, Connolly AM, Yu X, Wickline SA: Occult cardiac contractile dysfunction in dystrophindeficient children revealed by cardiac magnetic resonance strain imaging. Circulation 2005, 112:2462-2467.

9. Hor KN, Wansapura J, Markham LW, Mazur W, Cripe LH, Fleck R, Benson DW, Gottliebson WM: Circumferential strain analysis identifies strata of cardiomyopathy in Duchenne muscular dystrophy: a cardiac magnetic resonance tagging study. J Am Coll Cardiol 2009, 53:1204-1210.

10. Bottomley PA, Hardy CJ, Argersinger RE, Allen-Moore G: A review of $1 \mathrm{H}$ nuclear magnetic resonance relaxation in pathology: are $\mathrm{T} 1$ and $\mathrm{T} 2$ diagnostic?. Med Phys 1987, 14:1-37.

11. Bottomley PA, Foster TH, Argersinger RE, Pfeifer LM: A review of normal tissue hydrogen NMR relaxation times and relaxation mechanisms from 1-100 MHz: dependence on tissue type, NMR frequency, temperature, species, excision, and age. Med Phys 1984, 11:425-448.

12. Huang Y, Majumdar S, Genant HK, Chan WP, Sharma KR, Yu P, Mynhier M, Miller RG: Quantitative MR relaxometry study of muscle composition and function in Duchenne muscular dystrophy. J Magn Reson Imaging 1994, 4:59-64.

13. Boxt LM, Hsu D, Katz J, Detweiler P, McLaughlin S, Kolb TJ, Spotnitz HM: Estimation of myocardial water content using transverse relaxation time from dual spin-echo magnetic resonance imaging. Magn Reson Imaging 1993, 11:375-383.

14. Takeuchi M, Sekino M, Iriguchi N, Ueno S: Dependence of the spin-spin relaxation time of water in collagen gels on collagen fiber directions. Magn Reson Med Sci 2004, 3:153-157.

15. Choi SH, Kang JW, Kim ST, Lee BH, Chun EJ, Schuleri KH, Choi SI, Lim TH: Investigation of T2-weighted signal intensity of infarcted myocardium and its correlation with delayed enhancement magnetic resonance imaging in a porcine model with reperfused acute myocardial infarction. Int J Cardiovasc Imaging 2009, 25(Suppl 1):111-119.

16. Cury RC, Shash K, Nagurney JT, Rosito G, Shapiro MD, Nomura CH, Abbara S, Bamberg F, Ferencik M, Schmidt EJ, Brown DF, Hoffmann U, Brady TJ: Cardiac magnetic resonance with T2-weighted imaging improves detection of patients with acute coronary syndrome in the emergency department. Circulation 2008, 118:837-844.

17. Stork A, Muellerleile K, Bansmann PM, Graessner J, Kaul M, Kemper J, Adam G, Lund GK: Value of T2-weighted, first-pass and delayed enhancement, and cine CMR to differentiate between acute and chronic myocardial infarction. Eur Radiol 2007, 17:610-617.

18. Zagrosek A, Abdel-Aty H, Boye P, Wassmuth R, Messroghli D, Utz W, Rudolph A, Bohl S, Dietz R, Schulz-Menger J: Cardiac magnetic resonance monitors reversible and irreversible myocardial injury in myocarditis. JACC Cardiovasc Imaging 2009, 2:131-138.

19. Shen $C T$, Jeng $C M$, Lin YM, Chieng PU: Intensification of relative myocardial T2-weighted magnetic resonance signals in patients with acute viral myocarditis: report of one case. Zhonghua Min Guo Xiao Er Ke Yi Xue Hui Za Zhi 1993, 34:405-411.

20. Marie PY, Carteaux JP, Escanye JM, Claudon O, David N, Mattei S, Hassan N, Danchin N, Karcher G, Bertrand A, Villemot JP: Detection and prediction of acute heart transplant rejection with the myocardial $\mathrm{T} 2$ determination provided by a black-blood magnetic resonance imaging sequence. $J$ Heart Lung Transplant 2001, 20:193-194.

21. Mavrogeni S, Tzelepis GE, Athanasopoulos G, Maounis T, Douskou M, Papavasiliou A, Cokkinos DV: Cardiac and sternocleidomastoid muscle involvement in Duchenne muscular dystrophy: an MRI study. Chest 2005, 127:143-148.

22. Mavrogeni S, Papavasiliou A, Douskou M, Kolovou G, Papadopoulou E, Cokkinos DV: Effect of deflazacort on cardiac and sternocleidomastoid muscles in Duchenne muscular dystrophy: a magnetic resonance imaging study. Eur J Paediatr Neurol 2009, 13:34-40.

23. Kissoon N, Hor KN, Wansapura JP, Mazur W, Fleck RJ, Puchalski MD, Benson DW, Gottliebson WM: Increased susceptibility of the left lateral free wall to myocardial delayed enhancement in Duchenne Muscular Dystrophy: progressive systolic dysfunction demonstrable by CMR regional strain analysis. Journal of Cardiovascular Magnetic Resonance 2009, 11:p12.

24. Puchalski MD, Williams RV, Askovich B, Sower CT, Hor KH, Su JT, Pack N, Dibella $E$, Gottliebson WM: Late gadolinium enhancement: precursor to cardiomyopathy in Duchenne muscular dystrophy?. Int I Cardiovasc Imaging 2009, 25:57-63.

25. Loganathan R, Bilgen M, Al-Hafez B, Smirnova IV: Characterization of alterations in diabetic myocardial tissue using high resolution MRI. Int $J$ Cardiovasc Imaging 2006, 22:81-90.

26. Nishimura T, Yanagisawa A, Sakata H, Sakata K, Shimoyama K, Ishihara T, Yoshino H, Ishikawa K: Thallium-201 single photon emission computed tomography (SPECT) in patients with duchenne's progressive muscular dystrophy: a histopathologic correlation study. Jpn Circ J 2001, 65:99-105.

27. Silva C, Moon JC, Elkington AG, John AS, Mohiaddin RH, Pennell DJ: Myocardial late gadolinium enhancement in specific cardiomyopathies by cardiovascular magnetic resonance: a preliminary experience. $J$ Cardiovasc Med (Hagerstown) 2007, 8:1076-1079.

28. Varghese A, Pennell DJ: Late gadolinium enhanced cardiovascular magnetic resonance in Becker muscular dystrophy. Heart 2004, 90:e59.

29. Yilmaz A, Gdynia HJ, Baccouche H, Mahrholdt H, Meinhardt G, Basso C, Thiene G, Sperfeld AD, Ludolph AC, Sechtem U: Cardiac involvement in patients with Becker muscular dystrophy: new diagnostic and pathophysiological insights by a CMR approach. J Cardiovasc Magn Reson 2008, 10:50.

30. Giri S, Chung YC, Merchant A, Mihai G, Rajagopalan S, Raman SV, Simonetti OP: T2 quantification for improved detection of myocardial edema. J Cardiovasc Magn Reson 2009, 11:56.

31. Bendall MR, Garwood M, Ugurbil K, Pegg DT: Adiabatic refocusing pulse which compensates for variable rf power and off-resonance effects. Magn Reson Med 1987, 4:493-499.

32. Garwood M, Ugurbil K, Rath AR, Bendall MR, Ross BD, Mitchell SL, Merkle H: Magnetic resonance imaging with adiabatic pulses using a single surface coil for RF transmission and signal detection. Magn Reson Med $1989,9: 25-34$

33. Hwang TL, van Zijl PC, Garwood M: Fast broadband inversion by adiabatic pulses. J Magn Reson 1998, 133:200-203.

34. Bartlett PA, Symms MR, Free SL, Duncan JS: T2 relaxometry of the hippocampus at 3T. AJNR Am J Neuroradiol 2007, 28:1095-1098.

doi:10.1186/1532-429X-12-14

Cite this article as: Wansapura et al:: Left ventricular T2 distribution in Duchenne Muscular Dystrophy. Journal of Cardiovascular Magnetic Resonance 2010 12:14.

\section{Submit your next manuscript to BioMed Central and take full advantage of:}

- Convenient online submission

- Thorough peer review

- No space constraints or color figure charges

- Immediate publication on acceptance

- Inclusion in PubMed, CAS, Scopus and Google Scholar

- Research which is freely available for redistribution
C Biomed Central 